\title{
Mass Scale Artemisinin Production in a Stirred Tank Bioreactor Using Hairy Roots of Artemisia Annua
}

\author{
Nivedita Patra ${ }^{1,2}$, Ashok Kumar Srivastava ${ }^{1^{*}}$ \\ 1Department of Biochemical Engineering and Biotechnology, IIT Delhi, Hauz Khas, New Delhi - 110016, \\ India. \\ 2Department of Biotechnology and Medical Engineering, National Institute of Technology Rourkela, Odisha, \\ India - 769008. \\ * Corresponding author. Tel.: 26591010, email: ashokks@dbeb.iitd.ac.in \\ Manuscript submitted June 11, 2014; accepted August 18, 2014. \\ doi: 10.17706/ijbbb.2014.4.6.467-474
}

\begin{abstract}
Abastract: Presently, artemisinin is isolated from the shoots of artemisia annua plant. However, due to seasonal availability of the plant, the supply of this drug is much lower than its demand. Alternative biotechnological protocols are, therefore, highly needed to supplement the supply of this drug. One such production protocol could be mass scale hairy root cultivations in carefully identified bioreactor. Hairy root were induced by the Agrobacterium rhizogenes mediated genetic transformation in vitro grown plants of Artemisia annua. The hairy root propagation conditions were thereafter optimized to enhance the biomass and artemisinin accumulation which resulted in following cultivation and environmental conditions rotational speed $(70 \mathrm{rpm})$, temperature $\left(25^{\circ} \mathrm{C}\right)$, size of inoculum $(1 \mathrm{~g} / \mathrm{l} \mathrm{DW})$, age of inoculum (8 d) and medium to vessel volume ratio $(0.18)$. Scale-up of the hairy root cultivation was thereafter performed in the stirred tank bioreactor for mass scale artemisinin production in an appropriate bioreactor configuration. Hairy root cultivation in stirred tank bioreactor cultivation resulted not only in high biomass accumulation of $6.3 \mathrm{~g} / \mathrm{l}$ dry weight (37.50 g fresh weight) but also artemisinin content of $0.32 \mathrm{mg} / \mathrm{g}$ by using optimized media after $25 \mathrm{~d}$ of batch cultivation.
\end{abstract}

Key words: Artemisia annua, artemisinin, hairy root culture, optimization, bioreactor, biomass.

\section{Introduction}

Treatment of malaria is normally done by anti-malarial drugs like quinine. The emergence of multiple drug-resistant strains of Plasmodium has resulted in different severe forms of malaria, like cerebral malaria, which cannot be cured by quinine.

Artemisinin and its derivatives emerged as a useful class of drugs, which are effective against all known strains of the deadly parasite when used in combination with quinine, so that there have been no reports of artemisinin resistance with the malarial parasite. Artemisinin is isolated from the seasonal herb plant Artemisia annua. However the content of artemisinin is low in Artemisia annua and it varies from $0.04 \%$ to $1 \%$ depending on the cultivar as well as other cultivation conditions like temperature, availability of nutrients etc. [1]. The artemisinin content in field propagated plantations are subject to seasonal variations, and variability in the different generations due to cross-pollination, which results in less artemisinin production and productivity. Therefore, in vitro production protocols may supplement the production of large amount of important plant products. Plant cell/ hairy root cultivation has been demonstrated as a potent alternative for the production of plant metabolites [2] and for artemisinin accumulation as well [3]. 
Successful large scale cultivation of plant cell/hairy root under in vitro conditions could be a major protocol for commercialization of artemisinin production via this production route. Plant cells have some inherent disadvantages, e.g. shear sensitivity and genetic instability over a period of time, while hairy roots are biochemically more stable and can grow in a hormone free media.

The mass production of artemisinin by using hairy root culture technologies is still in nascent stage, however some investigations have been successful in the induction / propagation and maintenance of hairy root cultures under in vitro conditions for more than 15 years [3], [4]. Some lab scale bioreactor studies have also been reported but were not able to produce reasonably high amounts of artemisinin [5]. However, the feasibility of large scale production of artemisinin by hairy root cultivation using a suitable bioreactor system has several challenges, primarily, due to the complex structure of hairy roots, slow growth $(>2-3$ weeks cultivation time, $>12 \mathrm{~h}$ doubling time), complex genetic control of secondary metabolite production, non-uniform growth, difficulty in estimation of biomass concentration, shear sensitive nature of hairy roots, mass transfer limitation due to heterogeneous growth in bioreactors particularly having no impellers [5]. For artemisinin production, liquid-phase reactor like bubble column reactor has been reported to be better for high biomass accumulation [6]. In this study, liquid phase bioreactor configurations i.e. bubble column reactor was utilized for the mass-scale hairy root cultivation and its suitability for commercial use was established.

\section{Material and Methods}

\subsection{Induction of Hairy Roots}

Induction of hairy roots was done according to the protocol reported in literature [7] with minor modifications as described below- The explants of A. annua were germinated. The leaves of the in vitro grown plants were then incubated on solid plates of MS media for $24 \mathrm{~h}$. These precultured explants were then infected with exponentially growing culture of Agrobacterium rhizogenes strain LBA 301 which was facilitated by dipping the cut ends of apical meristem explants in bacterial cultures for $20 \mathrm{~min}$. The infected explants were then transferred to MS agar media flasks and incubated at $25{ }^{\circ} \mathrm{C}$ for $2 \mathrm{~d}$ in dark. Agrobacterium infection was, thereafter, removed by transferring explants to MS-Agar medium and antibiotic solutions (Cefotaxime $500 \mathrm{mg} / \mathrm{l}$ ) for 2 subculture cycles. The hairy roots emerging from the cut ends of plant tissue were allowed to grow till it was about $4 \mathrm{~cm}$ in length. These were then aseptically excised from the parent plant and subcultured in MS media for the propagation of parent cell line which was, thereafter, subcultured in MS media every three weeks for routine maintenance.

\subsection{Optimization of Hairy Root Cultivation Conditions in Shake Flask}

\subsubsection{Effect of shear stress}

In this study, $0.1 \mathrm{~g}$ (on DW basis) Artemisia annua hairy roots were aseptically transferred to $500 \mathrm{ml}$ Erlenmeyer flask containing $100 \mathrm{ml}$ MS media. Five identical flasks were prepared (in duplicate) for the study of shear stress. These hairy root shake flask cultures were incubated at $25{ }^{\circ} \mathrm{C}$ under $16 / 8 \mathrm{~L} / \mathrm{D}$ photoperiod regime on a gyratory shaker maintained at different rotational speeds $(60,70,80,100$ and 120 $\mathrm{rpm})$. The roots were harvested after $15 \mathrm{~d}$ cultivation period.

\subsubsection{Effect of temperature}

Artemisia annua hairy roots ( $0.1 \mathrm{~g}$ (on DW basis)) were aseptically transferred to $500 \mathrm{ml}$ Erlenmeyer flask containing $100 \mathrm{ml}$ MS media. Four identical flasks (in duplicate) were prepared for the study of effect of temperature on response of hairy roots. These hairy root shake flask cultures were then incubated at an agitation speed of $70 \mathrm{rpm}$ under 16/8 L/D photoperiod regime on a gyratory shaker set at different temperatures $\left(20,25,30\right.$ and $\left.35^{\circ} \mathrm{C}\right)$. The roots were harvested after $15 \mathrm{~d}$ cultivation period. 


\subsubsection{Effect of size of inoculum}

Artemisia annua hairy roots were aseptically transferred to $500 \mathrm{ml}$ Erlenmeyer flask containing $100 \mathrm{ml}$ MS media. The size of inoculum in the hairy root culture was varied at different concentrations $(0.01,0.02$, $0.05,0.10,0.12,0.15 \mathrm{~g}$ per $100 \mathrm{ml}$ of medium on DW basis). Six identical flasks (in duplicate) were prepared in order to study the effect of inoculum size on biomass and artemisinin accumulation. These hairy root shake flask cultures were incubated at $25^{\circ} \mathrm{C}$ under 16/8 L/D photoperiod regime on a gyratory shaker maintained at rotational speed of $70 \mathrm{rpm}$. The roots were harvested after $15 \mathrm{~d}$ of cultivation period.

\subsubsection{Effect of age of inoculum}

The inoculum for initiating hairy root culture of Artemisia annua in shake flasks was developed by cultivating the roots in MS media. After different time periods of growth (8d, $15 \mathrm{~d}, 20 \mathrm{~d}, 30 \mathrm{~d}$ ) in MS media $0.1 \mathrm{~g}$ of hairy root inoculum were aseptically transferred to $500 \mathrm{ml}$ Erlenmeyer flask containing $100 \mathrm{ml} \mathrm{MS}$ media. Four identical flasks were prepared (in duplicate) to study the effect of age of inoculum. These hairy root shake flask cultures were incubated at $25^{\circ} \mathrm{C}$ under $16 / 8 \mathrm{~L} / \mathrm{D}$ photoperiod regime on a gyratory shaker maintained at rotational speed of $70 \mathrm{rpm}$. The roots were harvested after $15 \mathrm{~d}$ cultivation period.

\subsection{Optimization of Medium Volume to Total Shake Flask Volume (Vm/Vf) Ratio}

The total volume of cultivation medium in the shake flask was altered to establish different medium volume $\left(V_{\mathrm{m}}\right)$ to total shake flask volume $\left(V_{\mathrm{f}}\right)$. The impact of different $\left(V_{\mathrm{m}} / V_{\mathrm{f}}\right)$ ratios $(0.06,0.12,0.15,0.18$, 0.24 ) on biomass and artemisinin accumulation was observed. Artemisia annua hairy roots $(1 \mathrm{~g} / \mathrm{l} \mathrm{DW}$ basis $)$ were aseptically transferred to $500 \mathrm{ml}$ shake flask containing the MS media (as required for different $V_{\mathrm{m}} / V_{\mathrm{f}}$ ratio). The different shake flasks were incubated at $25{ }^{\circ} \mathrm{C}$ under $16 / 8 \mathrm{~h} \mathrm{~L} / \mathrm{D}$ photoperiod for $15 \mathrm{~d}$ on a gyratory shaker maintained at $70 \mathrm{rpm}$.

\subsection{Cultivation in Stirred Tank Reactor}

A 3-l stirred tank reactor (Applikon Dependable Instruments, The Netherlands) with custom made setric impeller (for low shear) was initially used for the mass cultivation of hairy roots. Artemisinin biosynthetic pathway was indicated to be light and oxygen dependent, therefore special provision for white fluorescent light was done for the bioreactor. $40 \% \mathrm{v} / \mathrm{v}$ oxygen was supplied along with ambient air in the liquid-phase bioreactors to manipulate the fraction of $\mathrm{O}_{2}$ in the air. $1 \mathrm{~g} / \mathrm{l}$ hairy root inoculum (on DW basis) was aseptically transferred to the bioreactors. The temperature was maintained at $25{ }^{\circ} \mathrm{C}$. Statistically optimized medium was used for cultivation of Artemisia annua hairy roots which consisted of nitrate/ ammonium ratio: $3.5, \mathrm{KH}_{2} \mathrm{PO}_{4}: 0.5 \mathrm{mM}$, sucrose: $37.134 \mathrm{~g} / \mathrm{l}$, GA3: $10 \mu \mathrm{g} / \mathrm{l}$ in MS/4 medium. The $\mathrm{pH}$ of media was maintained at 5.8 \pm 0.1 using Biocontroller (Applikon Dependable Instruments, The Netherlands). The rotational speed of setric impeller in the reactor was kept constant at $125 \mathrm{rpm}$ throughout the cultivation.

\section{Results and Discussion}

\subsection{Induction of Hairy Roots}

Induction of hairy roots was done as described earlier in previous sections. The protocol of induction of the hairy roots significantly affected the genetic transformation efficiency. The two commonly used transformation techniques are - either by wounding, inoculating of the explants nor by co-cultivation with the bacterial culture. Some of the trials attempted in this investigation are described below. In one of the induction procedure explants (leaves and apical meristem) were pricked with a needle of a syringe containing Agrobacterium rhizogenes culture $(5 \mathrm{ml})$ and submerged in the bacterial culture (to increase the probability of successful infection) for $5 \mathrm{~min}$. The bacterial solution was removed after $20 \mathrm{~min}$. The plant part was dried on sterilized filter paper and thereafter, incubation of bacterial infected plant part was done 
in petriplates (containing MS solid media) incubated in Castor Racks at $25^{\circ} \mathrm{C}$ having $16 / 8 \mathrm{~h} \mathrm{~L} / \mathrm{D}$ photo cycle. It was observed that hairy root induction was monitored. Using this procedure, no hairy roots were induced presumably due to excessive stress to the cut ends of plant parts.

In another procedure the sterilized explants (apical meristem, leaf) were dipped in the saturated bacterial cultures for $20 \mathrm{~min}$ so that the Agrobacterium could possibly infect the plant cells from the cut ends. No pricking with needles was done in this method. Rest of the procedure was same as that described above. Hairy roots emerged from the infected cut ends of explants confirming successful genetic transformation event using the co-cultivation technique. The clear difference in susceptibility of Artemisia annua to the two techniques indicates the complex genotype-strain interaction due to exogenous and endogenous rol gene products present in Agrobacterium cultures.

The initiated roots were allowed to grow with the mother explants till the length of the root was at least 4 $\mathrm{cm}$ and preferably profuse branching started. The roots were excised from the explants and transferred to fresh MS media containing $500 \mathrm{mg} / \mathrm{l}$ of the broad-spectrum antibiotic (Cephotaxime). No bacterial growth was observed after 3 subculture cycles in antibiotic containing MS media. When the hairy roots grown was atleast $2 \mathrm{~cm}$ long roots were subcultured as independent root lines in $50 \mathrm{ml}$ liquid MS medium. The hairy root lines were subcultured at an interval of $20 \mathrm{~d}$.

\subsection{Optimization of Shake Flask Cultivation Conditions}

\subsubsection{Effect of shear stress}

The effect of increasing rotational speed on biomass (and growth index) was investigated and established. The growth index increased with increasing rpm till $70 \mathrm{rpm}$. This could be due to better oxygen and nutrient transfer at high agitation rate during the shake flask cultivations [4]. Maximum biomass production of $3.64 \pm 0.39 \mathrm{~g} / \mathrm{l}$ and $20 \mu \mathrm{g} / \mathrm{g}$ DW artemisinin was obtained at atational speed of $70 \mathrm{rpm}$. Increasing the rpm beyond $70 \mathrm{rpm}$ led to decreased cell viability due to severe shear stress. When rpm lower than 70 the growth index was decreased primarily due to low solubility of oxygen and poor mixing which lead to leaking of toxic byproducts and intra-cellular pigments under those conditions.

\subsubsection{Effect of temperature}

The hairy root cultivation was initiated in the shake flasks in optimum cultivation conditions and media. The hairy root growth was continued for $15 \mathrm{~d}$ at 4 different temperature conditions $\left(20,25,30,35^{\circ} \mathrm{C}\right)$. The growth index at each temperature is summarised in Table 1 . The best hairy root growth $(3.56 \pm 0.15 \mathrm{~g} / \mathrm{l})$ and the growth index $2.56 \pm 0.15$ were obtained at $25^{\circ} \mathrm{C}$. The specific growth rate depends on the temperature of hairy root cultivation. High growth rate has been observed at $25^{\circ} \mathrm{C}$ whereas at $35{ }^{\circ} \mathrm{C}$, the specific growth rate becomes zero or the hairy root growth deteriorated and finally it died. It was further observed that as the temperature increases the probability of death of root tips increases. At a very low temperature the branching time of hairy roots become very high with the result growth rate was slowed down.

Table 1. Effect of Temperature on Growth Index of Artemisia Annua Hairy Root Culture

\begin{tabular}{llll}
\hline \hline S. No & Temperature $\left({ }^{\circ} \mathrm{C}\right)$ & Biomass $(\mathrm{g} / \mathrm{l})$ & Growth index \\
\hline 1 & 20 & $1.93 \pm 0.52$ & $0.93 \pm 0.52$ \\
2 & 25 & $3.56 \pm 0.15$ & $2.56 \pm 0.15$ \\
3 & 30 & $2.39 \pm 0.60$ & $1.39 \pm 0.60$ \\
4 & 35 & $0.61 \pm 0.05$ & 0 \\
\hline \hline
\end{tabular}

\subsubsection{Effect of size of inoculum}

The optimization of the size of inoculum provides a uniform basis of comparison of growth as well as secondary metabolite production. The hairy root cultivation shake flasks were inoculated with different inoculum size of hairy roots $(0.11,0.34,0.71,1.0,1.3,1.6 \mathrm{~g} / \mathrm{l} \mathrm{DW}$ basis $)$ and incubated for $15 \mathrm{~d}$ to elucidate 
the effect of size of inoculum on hairy root biomass growth. It was observed that at very low inoculum size $(0.11 \mathrm{~g} / \mathrm{l} \mathrm{DW})$ the growth index was high (7.72) but the final biomass accumulated was far less $(0.96 \mathrm{~g} / \mathrm{l})$ than maximum biomass $(5.67 \pm 0.41 \mathrm{~g} / \mathrm{l})$ which was accumulated when hairy root cultivation was initiated with inoculum of $1 \mathrm{~g} / \mathrm{l}$ (on DW basis). A decrease in growth index on increasing the inoculum size beyond 1 g/l DW basis was observed presumably due to poor nutrient and/or oxygen availability for cultivations at very high inoculum size ( $1.5 \mathrm{~g} / \mathrm{l}$ inoculum). The effect of the size of inoculum used on initiation of hairy root culture is summarized in Table 2.

\begin{tabular}{cllll}
\multicolumn{5}{c}{ Table 2. Effect of Size of Inoculum on Growth of Hairy Root Culture of ARTEMISIA Annua } \\
\hline \hline S. No. & $\begin{array}{c}\text { Inoculum Size } \\
\text { (g/l, DW) }\end{array}$ & $\begin{array}{l}\text { Biomass } \\
\text { (g/l, DW) }\end{array}$ & $\begin{array}{l}\text { Growth index } \\
(\mathrm{g} / \mathrm{g})\end{array}$ & $\begin{array}{c}\text { Average growth rate } \\
(\mathrm{GR})(\mathrm{d}-1)\end{array}$ \\
\hline 1) & 0.11 & 0.96 & 7.72 & 0.51 \\
$2)$ & 0.34 & 2.08 & 5.11 & 0.34 \\
$3)$ & 0.71 & 3.76 & 4.29 & 0.28 \\
$4)$ & 1.0 & 5.67 & 4.67 & 0.31 \\
$5)$ & 1.3 & 3.19 & 1.45 & 0.10 \\
$6)$ & 1.6 & 4.29 & 1.68 & 0.11 \\
\hline \hline
\end{tabular}

\subsubsection{Effect of age of inoculum}

The age of inoculum also turned out to be a crucial factor for accumulation of artemisinin in hairy roots. Hairy root cultures growing at different stages of log and stationary phase of cultivation were used as the inoculum for initiation of hairy roots in shake flask cultures. Of all the inoculum stage (8-30 d) studied 8 day old, the inoculum was found to be optimal for maximum growth of hairy roots $(5.67 \pm 0.41 \mathrm{~g} / \mathrm{l}$ biomass $)$ and artemisinin accumulation $(0.10 \mathrm{mg} / \mathrm{g})$. On increasing the age of inoculum further poor growth index $(3.19 \pm 0.12 \mathrm{~g} / \mathrm{l}$ for $30 \mathrm{~d}$ inoculum age) was observed. In literature it has been observed that artemisinin content varies with the culture age as well as age of inoculum used for initiation of hairy root cultivation [8].

\subsection{Optimization of Medium Volume to Total Shake Flask Volume (Vm/ Vf) Ratio}

While optimizing the medium volume to flask volume ratio $\left(V_{\mathrm{m}} / V_{\mathrm{f}}\right)$, it was observed that the biomass accumulation increased from $2.38 \mathrm{~g} / \mathrm{l}$ at $V_{\mathrm{m}} / V_{\mathrm{f}}$ of 0.06 to a reasonably high value of $5.72 \mathrm{~g} / \mathrm{l}$ at a $V_{\mathrm{m}} / V_{\mathrm{f}}$ ratio of 0.18 , which thereafter, decreased to $4.6 \mathrm{~g} / \mathrm{l}$ at an increased $V_{\mathrm{m}} / V_{\mathrm{f}}$ ratio of 0.24 (Fig. 1). The increase in the hairy root biomass growth while decreasing the $V_{\mathrm{m}} / V_{\mathrm{f}}$ ratio from 0.24 to 0.18 could be supported by the fact that a reduction in the medium to flask volume ratio $\left(V_{\mathrm{m}} / V_{\mathrm{f}}\right)$ changes the oxygenation of shake flask hairy root cultures significantly because it increases the gas-liquid interfacial area. In above studies it was also observed that the artemisinin accumulation (content) in the hairy roots continuously increased from 1.11 $\mathrm{mg} / \mathrm{l}$ to $1.49 \mathrm{mg} / \mathrm{l}$ with an increase in the $V_{\mathrm{m}} / V_{\mathrm{f}}$ ratio (from 0.06 to 0.18 ). In order to achieve maximum overall volumetric productivity (mg/ (l.d)) of artemisinin in $15 \mathrm{~d}$ of the hairy root cultivation period. It was necessary to calculate the maximum artemisinin production $(\mathrm{mg} / \mathrm{l})$, which was essentially a cumulative effect of the biomass production $(\mathrm{g} / \mathrm{l})$ and the artemisinin accumulation within the hairy roots $(\mathrm{mg} / \mathrm{g})$. As can be observed from Fig. 1, highest artemisinin production $(1.49 \mathrm{mg} / \mathrm{l})$ was achieved at $\left(V_{\mathrm{m}} / V_{\mathrm{f}}\right)$ the ratio of 0.18 in $15 \mathrm{~d}$ of the hairy root cultivation period. Hence the optimum value of the medium to flask volume ratio $\left(V_{\mathrm{m}} / V_{\mathrm{f}}\right)$ for the initiations of the hairy root liquid culture was identified as 0.18 . It has been reported in literature that the mass transfer between the gas and liquid phase occurs only at the walls of the agitating shake flasks [9]. Hairy roots decrease the motion of fluid in the shake flasks. Optimal growth occurs when the rate of oxygen transfer is equal to or higher than the oxygen uptake rate of the hairy roots.

From above independent studies, it conducted so far the optimum physical parameters were identified and summarized in Table 3. 


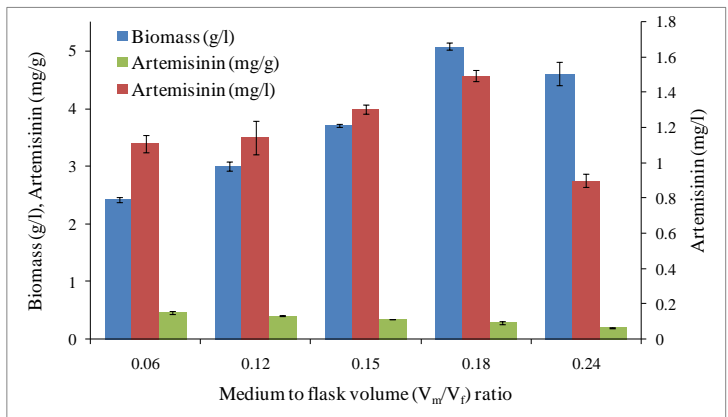

Fig. 1. Effect of medium to flask volume ratio on growth and artemisinin production in liquid culture of hairy roots.

Table 3. Optimum Values of Various Parameters of Hairy Root Culture in Shake Flask

\begin{tabular}{lll}
\hline \hline S. No & Variable & Optimum value \\
\hline 1 & Agitation speed $(\mathrm{rpm})$ & 70 \\
2 & Temperature $\left({ }^{\circ} \mathrm{C}\right)$ & 25 \\
3 & Size of inoculum $(\mathrm{g} / \mathrm{l} \mathrm{DW}$, basis) & 1.0 \\
4 & Age of inoculum $(\mathrm{d})$ & 8 \\
5 & $V_{\mathrm{m}} / V_{\mathrm{f}}$ ratio $(\mathrm{ml} / \mathrm{ml})$ & 0.18 \\
\hline \hline
\end{tabular}

\subsection{Cultivation of Hairy Roots in Stirred Tank Reactor}

Stirred tank bioreactor feature adequate, the availability of nutrient/oxygen during cultivations and is a preferred mode in microbial cultivation. Attempt was made to investigate the possibility by using STR for mass propagation of hairy roots for artemisinin accumulation. The cultivation however resulted in a good growth as shown in Fig. 2 (6.3 $\pm 0.45 \mathrm{~g} / \mathrm{l}$ of biomass was observed after $25 \mathrm{~d}$ cultivation). The hairy root cultivation accumulated $0.32 \mathrm{mg} / \mathrm{g}$ artemisinin on dry weight basis which was lesser than the shake flask cultivation conditions.

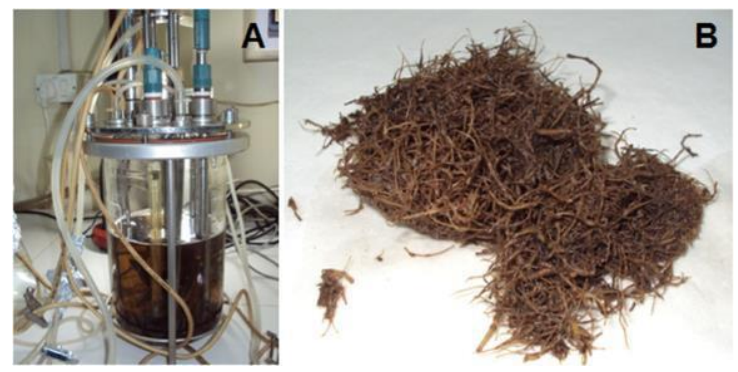

Fig. 2. Cultivation of hairy root culture in a 3-liter Stirred tank reactor. (A) Bioreactor set-up; (B) Hairy roots obtained after $25 \mathrm{~d}$ of growth.

\section{Conclusion}

The hairy root culture conditions were optimized in shake flask cultures of Artemisia annua. In order to ensure better oxygenation of shake flask hairy root cultures the volume of culture, medium to flask volume ratio $\left(V_{\mathrm{m}} / V_{\mathrm{f}}\right)$ also was studied and a $V_{\mathrm{m}} / V_{\mathrm{f}}$ ratio of 0.18 resulted in high biomass $(5.08 \mathrm{~g} / \mathrm{l})$ and artemisinin content $(1.49 \mathrm{mg} / \mathrm{l})$. Batch cultivation was, thereafter, performed for the hairy roots of Artemisia annua in a stirred tank reactor. The experiment was highly successful and resulted in a good growth yielding (6.3 \pm 0.45$)$ $\mathrm{g} / \mathrm{l}$ of biomass and an artemisinin content of $0.32 \mathrm{mg} / \mathrm{g}$ dry weight, obtained after $25 \mathrm{~d}$ cultivation.

\section{Acknowledgement}

The authors thankfully acknowledge the supply of elite seed material from CIMAP Lucknow. The financial 
support by Ministry of Human Resource Development, New Delhi (India) for the execution of the above project is gratefully acknowledged by one of the authors (Nivedita Patra).

\section{References}

[1] Delabays, N., Benakis, A., \& Collet, G. (1993). Selection and breeding for high artemisinin (qinghaosu) yielding strains of Artemsia annua. Acta. Hort., 330, 203-206.

[2] Srivastava, S., \& Srivastava, A. K. (2007). Hairy root culture for mass-production of high-value secondary metabolites. Crit. Rev. Biotechnol., 27, 29-43.

[3] Jaziri, M., Shimomura, K., Yoshimatsu, K., Fauconnier, M. L., Marlier, M., \& Homes, J. (1995). Establishment of Normal and Transformed Root Cultures of Artemisia annua L for Artemisinin Production, J. Plant Physiol., 145, 175-177.

[4] Weathers, P. J., Wyslouzil, B. E., Wobbe, K. K., Kim, Y. J., \& Yigit, E. (1999). The biological response of hairy roots to $\mathrm{O}_{2}$ levels in bioreactors. In vitro Cell. Dev. Biol. Plant, 35, 286-289.

[5] Kim, Y. J., Weathers, P. J., \& Wyslouzil, B. E. (2002a). Growth of Artemisia annua hairy roots in liquidand gas-phase reactors. Biotechnol. Bioeng., 80, 454-464.

[6] Kim, Y. J., Wyslouzil, B. E., \& Weathers, P. J. (2002b). Secondary metabolism of hairy root cultures in bioreactors. In Vitro Cell Dev. Biol. Plant, 38, 1-10.

[7] Putalun, W., Luealon, W., De-Eknamkul, W., Tanaka H., \& Shoyama, Y. (2007). Improvement of artemisinin production by chitosan in hairy root cultures of Artemisia annua L. Biotechnol. Lett., 29, 1143-1146.

[8] Weathers, P. J., Hemmavanh, D. D., Walcerz, D. B., Cheetham, R. D., \& Smith, T. C. (1997). Interactive effects of nitrate and phosphate salts, sucrose, and inoculum culture age on growth and sesquiterpene production in Artemisia annua hairy root cultures. In vitro Cell. Dev. Biol. Plant, 33, 306-312.

[9] Yu, S., \& Doran, P. M. (1994). Oxygen requirements and mass transfer in hairy-root culture. Biotechnol. Bioeng., 44, 880-887.

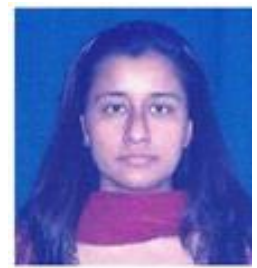

Nivedita Patra had done her M.Tech degree in biotechnology from the School of Biotechnology, GGSIPU, New Delhi. Ms. Patra is currently working as an assistant professor in the Biotechnology Department, National Institute of Technology Rourkela from 2014.

Currently she has also submitted her thesis for Ph.D. degree at the Department of Biochemical Engineering and Biotechnology, IIT Delhi on a topic entitled "Development and mass scale propagation of Artemisia annua hairy root culture in a suitable bioreactor system for Artemisinin production". She has presented several papers at national and international conferences and published 1 journal article. She has published 1 book chapter entitled "Statistical media optimization for enhanced biomass and Artemisinin production in Artemisia annua hairy roots" in a book entitled Chemistry of Phytopotentials: Health Energy and Environment Perspectives (Springer, Germany). She has also won the best poster prizes at international conference (CPHEE-2011 Agra) and IIT Delhi open house I2Tech-2012.

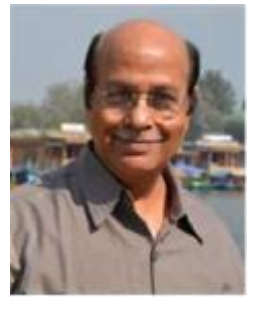

Ashok Kumar Srivastava received his Ph.D. degree from the McGill University, Montreal (Quebec) in 1990. He has extensive industrial research and teaching experience in the area of biochemical engineering and biotechnology. He has worked for about a year in the Sorbose fermentation plant of M/S Jayant Vitamins Limited, Ratlam.

He had worked in the Biochemical Engineering Group of National Chemical Laboratory, Pune for two years. He had served as a lecturer in the Biochemical 
Engineering and Food Technology Department of H.B. Technological Institute for ten years before joining IIT Delhi in 1991. He had received several awards and scholarships, the important ones among them are the first prizes for securing 1st position in B.Sc. in biochemical engineering and M.Tech. in biochemical engineering, National Overseas Scholarship award of MHRD, New Delhi for Ph.D. studies (1985-1990), UNESCO fellowship award (6 months) for advanced studies in Biochemical Engineering at Biochemical Engineering Group, Delft University, The Netherlands, "Biotechnology Overseas Associate ship" 3 month at Institut fur Technische Chemie, Hannover, Germany), "Biotechnology Overseas Associate ship" (3 month at Massey University, New Zealand). He is a life member of "Indian Institute of Chemical Engineers" and "Biotechnology Research Society of India". He is in the editorial board of Process Biochemistry and Journal of Food Science and Technology. 